RESEARCH ARTICLE

\title{
A New Model to Explain the Alignment of Certain Ancient Sites
}

\section{MARK J. CARLotTo}

Submitted July 19, 2019; Accepted September 4, 2019; Published June 30, 2020

https://doi.org/10.31275/2020/1619

Creative Commons License CC-BY-NC

\begin{abstract}
In a previous study of more than two hundred ancient sites, the alignments of almost half of the sites could not be explained. These sites are distributed throughout the world and include the majority of Mesoamerican pyramids and temples that are misaligned with respect to true north, megalithic structures at several sites in Peru's Sacred Valley, some pyramids in Lower Egypt, and numerous temples in Upper Egypt. A new model is proposed to account for the alignment of certain unexplained sites based on an application of Charles Hapgood's hypothesis that global patterns of climate change over the past 100,000 years could be the result of displacements of the Earth's crust and corresponding shifts of the geographic poles. It is shown that more than $80 \%$ of the unexplained sites reference four locations within $30^{\circ}$ of the North Pole that are correlated with Hapgood's hypothesized pole locations. The alignments of these sites are consistent with the hypothesis that if they were built in alignment with one of these former poles they would be misaligned to north as they are now as the result of subsequent geographic pole shifts.
\end{abstract}

Keywords: ancient sites; pyramid alignment; pole shifts

\section{INTRODUCTION}

In a previous study of ancient sites, the alignments of almost half of the sites could not be explained (Carlotto, 2020). These sites, which are distributed throughout the world, include the majority of Mesoamerican pyramids and temples that are misaligned with respect to true north, megalithic structures at several sites in Peru's Sacred Valley, some pyramids in Lower Egypt, and numerous temples in Upper Egypt. From a 
review of the archaeological and archaeoastronomical literature, eight basic reasons were identified that typically account for the orientation of an archaeological site: 1) to cardinal directions (i.e. facing north, south, east, and west), 2) to solstice sunrise or sunset directions, 3) to sunrise or sunset directions on days when the sun passes directly overhead, 4) to directions of major and minor lunar standstills, 5) to a planet, 6) to a star or constellation, 7) to magnetic north, and 8) in the direction of a site of religious or spiritual importance. We also considered other explanations such as landscape and topography that have been used in some cases to account for the alignment of certain sites. For example, Shaltout and Belmonte (2005) analyzed the orientation of more than one hundred temples in Upper Egypt and Lower Nubia to discover that they face many different directions. Their principal conclusion is that local topography (the course of the Nile), not astronomy, was the most important factor in aligning the foundations of the temples.

This paper proposes a new model to explain the alignment of certain sites throughout the world based on an application of Charles Hapgood's hypothesis that patterns of climate change over the past 100,000 years could be the result of displacements of the Earth's crust and corresponding shifts of the geographic poles. The next section discusses the origin of the idea of geographic pole shifts, how information about the motion of the geo-magnetic poles over time suggests that large shifts of geographic poles have occurred in the past, and possible relationships between geographic pole shifts and climate change. The following section describes a new model to explain the alignment of sites to previous pole locations based on an application and refinement of Hapgood's original pole shift hypothesis. Results are organized into eight geographic regions. It is shown that more than $80 \%$ of the unexplained sites in our previous study (Carlotto, 2020) reference at least one of these previous pole locations.

\section{GEOGRAPHIC POLE SHIFTS AND CLIMATE CHANGE}

Early in the 2oth century, Alfred Wegener and others theorized that the continents were once a single large landmass that broke up and slowly drifted apart. Wegener's theory of continental drift explained the complementary shape of coastlines-how the west coast of Africa seems to 
fit the east coast of the Americas—and the similarity in rock formations and fossils along matching coastlines. This theory, now known as plate tectonics, divides the outer layer of the crust, called the lithosphere, into a number of plates that move independently of one another over a less rigid layer called the asthenosphere (Kious \& Tilling, 1996). Holmes (1944) proposed that the Earth's mantle contains convection cells that dissipate interior heat and move the crust at the surface, thus providing a physical mechanism to drive plate motion. Inspired by Wegener's work, Milanković (1932) investigated the movement of the poles that he believed worked together with plate motion so that "the displacement of the pole takes place in such a way that ... Earth's axis maintains its orientation in space, but the Earth's crust is displaced on its substratum."

The earth's axis of rotation intersects the surface at the north and south geographic poles, which are currently located in the Arctic and Antarctic. The flow of liquid metal in the outer core generates electric currents. The rotation of earth on its axis causes these electric currents to induce a magnetic field. The location of the magnetic poles slowly wanders in a seemingly random manner around the geographic poles. Rocks, sediment, and archaeological artifacts that contain magnetic minerals such as magnetite record the direction and intensity of Earth's magnetic field when they are heated above the Curie temperature. When a paleomagnetic material cools, magnetic information is retained by the mineral grains. By collecting and analyzing samples at different times and in different places, it is possible to estimate the location of the magnetic poles (paleopoles) as a function of time.

Kirschvink et al. (1997) determined from paleomagnetic data collected in Australia and North America that a massive crustal shift occurred between 534 million and 505 million years ago, which caused Australia to rotate a quarter of the way around the globe. This shift occurred around the time of the Cambrian Explosion, when most groups of animals first appear in the fossil record, and is thought to have been a major factor in the evolutionary changes that later took place. Woodworth and Gordon (2018) used paleomagnetic and ocean sediment data to show that Greenland was much closer to the North Pole 12-48 million years ago than it is today. Daradich et al. (2017) estimate a steady shift of Earth's poles by $\sim 8^{\circ}$ over the last 40 million 
years toward Greenland has brought North America to increasingly higher latitudes and caused its climate to gradually cool over this period of time.

If polar motion affects climate, the converse may also be true. Prior to the year 2000, the North Pole was slowly moving toward Hudson Bay, at which time it changed direction and began to drift toward Greenland. Chen at al. (2013) claim that the change in direction was caused by the accelerated melting of the Greenland Ice Sheet. Adhikari and Ivins (2016) argue that polar motion is influenced by changes in the amount of water held within the continents. Although these factors appear to control the direction of polar motion, they do not appear sufficient to account for its magnitude. Adhikari et al. (2018) have come to the conclusion that mantle convection, which drives plate tectonics, also seems to be a significant factor affecting polar motion.

It is generally assumed that climate patterns are driven to a large extent by the amount of solar radiation reaching the Earth. The amount of radiation depends on a combination of factors including changes in the eccentricity in our orbit around the sun, axial tilt (obliquity), axial and apsidal precession, and orbital inclination. The combination of these effects gives rise to what are called Milanković cycles. Although there is extensive evidence that the variation in solar radiation is an important factor, there are certain problems with Milanković's model as it relates to the timing and magnitude of the cycles and their correlation with climate events. Muller and MacDonald (1997) suggest the possibility that an external factor such as extraterrestrial accretion of dust or meteoroids could affect climate. It has been hypothesized that the Younger Dryas period of rapid cooling in the late Pleistocene, 12,800 to 11,500 years ago, could have had an extraterrestrial cause such as the Taurid meteor swarm (Napier, 2010). Woelfli et al. (2002) propose that an encounter with a Mars-sized object at around this time moved the North Pole from Greenland to its present position.

\section{SHIFTED GEOGRAPHIC POLE SITE ALIGNMENT MODEL}

Hapgood (1958) hypothesized that climate changes and ice ages could be explained by large sudden shifts of the geographic pole. He cites extensive evidence suggesting that during the last ice age the North 
TABLE 1

Estimated Locations of the North Pole

\begin{tabular}{lcc}
\hline \hline Name & Latitude & Longitude \\
\hline Hudson Bay & $59.75^{\circ}$ & $-78.0^{\circ}$ \\
Greenland & $79.5^{\circ}$ & $-63.75^{\circ}$ \\
Norwegian Sea & $70.0^{\circ}$ & $-0^{\circ}$ \\
Bering Sea & $56.25^{\circ}$ & $-176.75^{\circ}$ \\
\hline
\end{tabular}

Pole was located at around $60^{\circ} \mathrm{N}, 83^{\circ} \mathrm{W}$, near Hudson Bay in Canada. Using climate data from a variety of sources, Hapgood reasoned that North America, which was then covered by a massive layer of ice and snow, was colder because it had been shifted closer to the pole, while places on the opposite side of the earth, such as Europe, were warmer because they had been shifted away from the pole and south toward the equator. By examining patterns of climate change, he estimated that three geographic pole shifts had taken place during the past 100,000 years: 1) from Hudson Bay $\left(60^{\circ} \mathrm{N} 73^{\circ} \mathrm{W}\right)$ to the current pole, 12,000 to 17,000 years ago, 2) from the Atlantic Ocean between Iceland and Norway $\left(72^{\circ} \mathrm{N} 10^{\circ} \mathrm{E}\right)$ to Hudson Bay, 50,000 to 55,000 years ago, and 3) from the Yukon $\left(63^{\circ} \mathrm{N} 135^{\circ} \mathrm{W}\right)$ to between Iceland and Norway, 75,000 to 80,000 years ago.

Rand Flem-Ath noted that if the North Pole were in Hudson Bay, the major axis of Teotihuacan, an ancient Mesoamerican city 25 miles northeast of modern-day Mexico City, which is currently oriented $15.4^{\circ}$ east of north, would be aligned to within a few degrees of due north (Wilson \& Flem-Ath, 2000). Motivated by this observation, more than fifty sites not aligned to north were identified that could have once been aligned to one of Hapgood's hypothesized pole locations. An algorithm was developed that used the orientation (azimuth) angle and geographic coordinates of these sites measured in Google Earth to estimate a set of hypothetical "best-fit" pole locations (Carlotto, 2019). Table 1 lists the four hypothetical locations of the North Pole computed by this algorithm. The estimated Hudson Bay pole location is less than 200 miles from Hapgood's original Hudson Bay pole. If the North Pole 


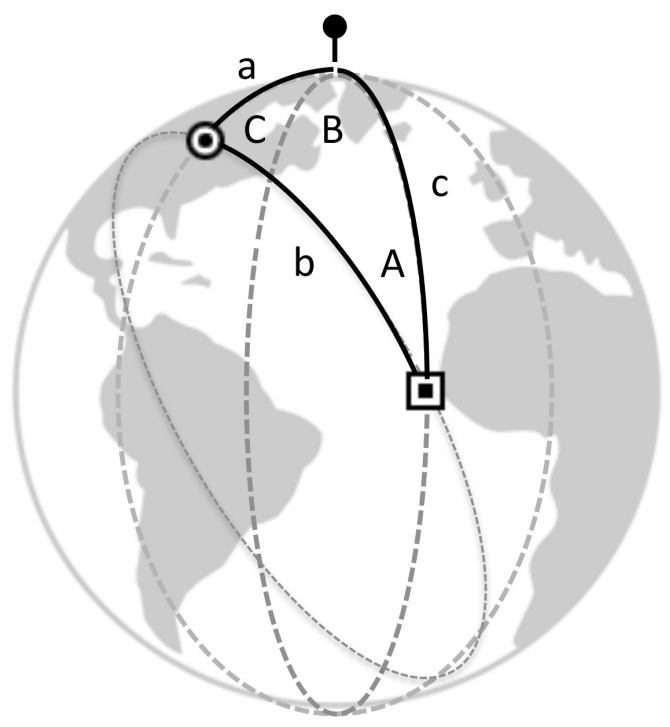

Figure 1. The locations of a site A, North Pole B, and previous pole C are the vertices of a spherical triangle. Segments of spherical triangles are great circles. The angle $A$ is the azimuth of the previous pole location measured at the site.

were at that location, Teotihuacan would be aligned to the cardinal directions. The estimated pole in northern Greenland is 1,250 miles west of Hapgood's original Iceland/Norway pole, and the estimated pole in the Norwegian Sea is about 300 miles south of it. A fourth computed pole location is in the Bering Sea north of the Aleutian Islands, about 1,500 miles from Hapgood's original Yukon pole.

With reference to Figure 1 , let $A$ be the location of a site, B the current location of the North Pole, and $C$ the location of the North Pole at the time the site was first established. The angle $A$ is the current alignment of the site with respect to north. A shift in the geographic pole causes both the latitude as well as the orientation of a site to change. If $\left(\lambda_{A}, \phi_{A}\right)$ and $\left(\lambda_{C}, \phi_{C}\right)$ are the latitudes and longitudes of the site and past pole in the current geographic reference frame, the orientation (rotation) angle of the site is

$$
A=\sin ^{-1}\left[\frac{\sin \mathrm{a} \sin \mathrm{B}}{\sqrt{1-(\cos a \cos c+\sin a \sin c \cos B)^{2}}}\right]
$$


where

$$
\begin{aligned}
& \alpha=\frac{\pi}{2}-\lambda_{c} \\
& c=\frac{\pi}{2}-\lambda_{A} \\
& B=\varphi_{C}-\varphi_{A}
\end{aligned}
$$

Its latitude prior to the pole shift would have been $90^{\circ}-\lambda_{C}$ where

$$
\lambda_{C}=\cos ^{-1}(\cos a \cos c+\sin a \sin c \cos B)
$$

By comparing the orientation angle of a site measured using Google Earth to Equation 1, it is possible to determine if the site could have once faced north. In addition, by substituting previous pole values from Equation 1 and Equation 3 into the solar and lunar alignment equations (Carlotto, 2020), it is possible to determine if the site was aligned to solstices, zenith passages, or lunar standstills relative to those poles.

\section{RESULTS: SITES ALIGNED TO PREVIOUS POLE LOCATIONS}

Tables 2-9 indicate the alignments for more than two hundred ancient sites to the current (Arctic Ocean) pole, and former estimated Hudson Bay, Greenland, the Norwegian Sea, and Bering Sea pole locations. The sites are organized into eight geographic regions. The key to the alignments is as follows:

Cardinal directions, i.e., geographic poles, and equinoxes (E)

Magnetic pole at the time of construction $(X)$

Zenith passage $(Z)$

Solstices (S)

Major and minor lunar standstills $(\mathrm{M}, \mathrm{m})$

Stellar alignments (st)

Alignments to "Sacred Directions" (D)

Only six of the eight alignment hypotheses were examined for the shifted poles, as there is insufficient information to evaluate "st", and "D" would not be affected by a crustal displacement. 
TABLE 2

\section{Alignments of Sites in Africa}

\begin{tabular}{|c|c|c|c|c|c|c|c|c|c|}
\hline Name & Latitude & Longitude & North & East & Current & Hudson Bay & Greenland & Norway Sea & Bering Sea \\
\hline Algeria, Jabal Lakhdar & 35.063404 & 1.183731 & -5 & 85 & & & & $\mathrm{E}$ & \\
\hline Egypt, Abu Rawash, Pyramid of Djedefre & 30.032262 & 31.074714 & 0 & 90 & $\mathrm{E}$ & & & & \\
\hline Egypt, Abusir, Pyramid of Neferefre & 29.89377 & 31.201454 & 0 & 90 & $\mathrm{E}$ & & & & \\
\hline Egypt, Abusir, Pyramid of Neferirkare & 29.895093 & 31.202249 & 0 & 90 & $\mathrm{E}$ & & & & \\
\hline Egypt, Abusir, Pyramid of Sahure & 29.897622 & 31.203367 & 0 & 90 & $\mathrm{E}$ & & & & \\
\hline Egypt, Abydon, Temple Ramses II & 26.186426 & 31.91628 & 44.2 & 134.2 & & & & $\mathrm{~s}$ & \\
\hline Egypt, Abydos, Osirion & 26.184099 & 31.918465 & 36.3 & 126.3 & & $\mathrm{~s}$ & & & \\
\hline Egypt, Abydos, Pyramid of Ahmose I & 26.175056 & 31.937822 & 36 & 126 & & $S$ & & & \\
\hline Egypt, Abydos, Temple Seti I & 26.184968 & 31.919183 & 36.3 & 126.3 & & $S$ & & & \\
\hline Egypt, Cairo, Mosque of Ibn Tulun & 30.028691 & 31.249394 & -39 & 51 & & & & & \\
\hline Egypt, Dahshur Pyramid of Senusret III & 29.818888 & 31.22555 & 0 & 90 & $\mathrm{E}$ & & & & \\
\hline Egypt, Dahshur, Bent Pyramid & 29.790449 & 31.209324 & 0 & 90 & $\mathrm{E}$ & & & & \\
\hline Egypt, Dahshur, Pyramid of Amenemhat II & 29.805807 & 31.223038 & 0 & 90 & $\mathrm{E}$ & & & & \\
\hline Egypt, Dahshur, Red Pyramid & 29.808882 & 31.206113 & 0 & 90 & $\mathrm{E}$ & & & & \\
\hline Egypt, Deir Bahari, Mortuary Temple of Mentuhotep II & 25.737375 & 32.606178 & 23.2 & 113.2 & S & & & & \\
\hline Egypt, Deir el Medinah, Temple of Hathor & 25.728846 & 32.602128 & -40 & 50 & & & $\mathrm{~s}$ & & \\
\hline Egypt, Dendara, Sacred Lake & 26.14180698 & 32.66953166 & 16.1 & 106.1 & & & & & $\mathrm{E}$ \\
\hline Egypt, Dendera, Temple of Hathor & 26.141914 & 32.670205 & 18.9 & 108.9 & st,m & & & S & \\
\hline Egypt, Edfu Temple of Horus & 24.976747 & 32.873087 & 12.8 & 102.8 & & & & $M$ & \\
\hline Egypt, Elephantine, Temple of Khnum & 24.084492 & 32.886206 & -42 & 48 & & & $M$ & & \\
\hline Egypt, Giza, Khafre & 29.975726 & 31.1308 & 0 & 90 & $\mathrm{E}$ & & & & \\
\hline Egypt, Giza, Khufu & 29.979067 & 31.13404 & 0 & 90 & $\mathrm{E}$ & & & & \\
\hline Egypt, Giza, Menkaure & 29.975811 & 31.131242 & 0 & 90 & $\mathrm{E}$ & & & & \\
\hline Egypt, Kom Ombo & 24.452085 & 32.928353 & 43.3 & 133.3 & & $\mathrm{~m}$ & & $\mathrm{~s}$ & \\
\hline Egypt, Lisht, Pyramid of Amenemhat I & 29.574802 & 31.225304 & 0 & 90 & $\mathrm{E}$ & & & & \\
\hline Egypt, Lisht, Pyramid of Senusret I & 29.56016 & 31.22113 & 0 & 90 & $E$ & & & & \\
\hline Egypt, Luxor West, Temple Ramses III & 25.719683 & 32.600711 & -42 & 48 & & & $\mathrm{M}$ & & \\
\hline Egypt, Luxor, Karnak, Temple of Amun Re & 25.718484 & 32.659044 & 26.6 & 116.6 & S & & & & \\
\hline Egypt, Meidum Pyramid & 29.388368 & 31.157503 & 0 & 90 & $\mathrm{E}$ & & & & \\
\hline Egypt, Pyramid of Teti & 29.875142 & 31.221847 & -12.5 & 77.5 & & & $\mathrm{E}$ & & \\
\hline Egypt, Saqqara, Mastaba of Shepseskaf & 29.838852 & 31.215273 & 0 & 90 & $\mathrm{E}$ & & & & \\
\hline Egypt, Saqqara, Pyramid of Djedkare-Isesi & 29.850983 & 31.220924 & 0 & 90 & $\mathrm{E}$ & & & & \\
\hline Egypt, Saqqara, Pyramid of Djoser & 29.87139735 & 31.21653162 & 5 & 95 & & & & & \\
\hline Egypt, Saqqara, Pyramid of Khendjer & 29.832363 & 31.224043 & 0 & 90 & E & & & & \\
\hline Egypt, Saqqara, Pyramid of Pepi II & 29.840246 & 31.213496 & 0 & 90 & E & & & & \\
\hline Egypt, Saqqara, Pyramid of Qakare Ibi & 29.84159 & 31.217712 & -10 & 80 & & & $E$ & & \\
\hline Egypt, Saqqara, Pyramid of Unas & 29.868182 & 31.215012 & 0 & 90 & $E$ & & & & \\
\hline Egypt, Saqqara, Pyramid Userkaf & 29.873332 & 31.219334 & 0 & 90 & $\mathrm{E}$ & & & & \\
\hline Egypt, Shunet El Zebib & 26.18951 & 31.908055 & -41.7 & 48.3 & & & & $\mathrm{~m}$ & \\
\hline Egypt, Siwa Oasis, Amun Temple & 29.201375 & 25.516151 & & & & & & & $\mathrm{E}$ \\
\hline Egypt, Temple of Edfu & 24.978092 & 32.873475 & 3 & 93 & & & & & \\
\hline Egypt, Temple of Esna & 25.29344448 & 32.55612504 & -23 & 67 & $\mathrm{M}$ & & & & \\
\hline Egypt, Temple of Hathor, El Kab & 25.138586 & 32.828651 & -44 & 46 & & & $\mathrm{M}$ & & \\
\hline Egypt, Temple of Isis at Shenhur & 25.86104 & 32.776808 & 10 & 100 & & & & M & \\
\hline Egypt, Temple of Ramses II & 25.727588 & 32.610283 & 41 & 131 & & & & $\mathrm{~S}$ & \\
\hline Egypt, Zawyet El Aryan, Layer Pyramid & 29.93282 & 31.161262 & -12 & 78 & & & $\mathrm{E}$ & & \\
\hline Ethiopia, Bete Giyorgis & 12.031714 & 39.04119 & 5.8 & 95.8 & $\mathrm{~m}$ & & & & \\
\hline Ethiopia, Yeha Temple & 14.28570335 & 39.01911389 & 11.4 & 101.4 & & & & $\mathrm{~m}$ & \\
\hline Sudan, Dangeil, Amun Temple & 18.131307 & 33.9598 & 16.5 & 106.5 & & & S & & $\mathrm{E}$ \\
\hline
\end{tabular}

$\mathrm{E}=$ cardinal directions, i.e. geographic poles and equinoxes. $\mathrm{M}, \mathrm{m}=$ major and minor lunar standstills. $S=$ solstices. st = stellar alignments. If no alignment is given, the reason is unknown. In some cases, there may be more than one explanation for an alignment. 
TABLE 3

\section{Alignments of Sites in Asia}

\begin{tabular}{|c|c|c|c|c|c|c|c|c|c|}
\hline Name & Latitude & Longitude & North & East & Current & Hudson Bay & Greenland & Norway Sea & Bering Sea \\
\hline Cambodia, Koh Ker & 13.78322 & 104.5374528 & -12.5 & 77.5 & $z$ & & & & \\
\hline Cambodia,Preah Khan of Kompong Svay & 13.40382 & 104.75421 & -28.2 & 61.8 & $M$ & & & & \\
\hline China, Chongling Mausoleum of Emperor Dezong of Tang & 34.70738 & 108.82853 & -4.2 & 85.8 & $x$ & & & & \\
\hline China, Jinling Mausoleum of Emperor Xianzong of Tang & 34.570992 & 108.265923 & -9 & 81 & $x$ & & & & \\
\hline China, The Lianhu Altar & 36.632869 & 101.746123 & 15.8 & 105.8 & s & & & & \\
\hline China, Tomb of Consort Ban & 34.379801 & 108.704492 & -11 & 79 & & & & & \\
\hline China, Tomb of Emperor Ai of Han & 34.400855 & 108.764606 & 0 & 90 & $E$ & & & & \\
\hline China, Tomb of Emperor Cheng of Han & 34.374896 & 108.698001 & -10 & 80 & $x$ & & & & \\
\hline China, Tomb of Emperor Gaozu of Han & 34.434691 & 108.876647 & -14 & 76 & & & & & \\
\hline China, Tomb of Emperor Hui of Han & 34.422895 & 108.841317 & -17 & 73 & & & & & \\
\hline China, Tomb of Emperor Jing of Han & 34.443823 & 108.940784 & 0 & 90 & E & & & & \\
\hline China, Tomb of Emperor Ping of Han & 34.397774 & 108.712421 & 0 & 90 & E & & & & \\
\hline China, Tomb of Emperor Wen of Sui & 34.28785 & 108.02289 & -3 & 87 & $x$ & & & & \\
\hline China, Tomb of Emperor Wu of Han & 34.338085 & 108.569684 & -8 & 82 & $x$ & & & & \\
\hline China, Tomb of Emperor Xuan of Han & 34.181063 & 109.022312 & 0 & 90 & $\mathrm{E}$ & & & & \\
\hline China, Tomb of Emperor Yuan of Han & 34.390303 & 108.739114 & 0 & 90 & $E$ & & & & \\
\hline China, Tomb of Emperor Zhao of Han & 34.361753 & 108.640108 & -11 & 79 & $x$ & & & & \\
\hline China, Tomb of Empress Dou & 34.235825 & 109.118614 & 22.6 & 112.6 & $\mathrm{~m}$ & & & & \\
\hline China, Tomb of Empress Dowager Bo & 34.220993 & 109.096341 & 21.6 & 111.6 & $\mathrm{~m}$ & & & & \\
\hline China, Tomb of Empress Fu & 34.402608 & 108.772545 & -4 & 86 & $x$ & & & & \\
\hline China, Tomb of Empress Li & 34.340327 & 108.562002 & -9.5 & 80.5 & $x$ & & & & \\
\hline China, Tomb of Empress Lü & 34.433824 & 108.881292 & -10.2 & 79.8 & s & & & & \\
\hline China, Tomb of Empress Shangguan & 34.363135 & 108.630538 & -8 & 82 & $x$ & & & & \\
\hline China, Tomb of Empress Wang (a) & 34.393242 & 108.733835 & 0 & 90 & $\mathrm{E}$ & & & & \\
\hline China, Tomb of Empress Wang (b) & 34.446291 & 108.9475 & 0 & 90 & $\mathrm{E}$ & & & & \\
\hline China, Tomb of Empress Wang (c) & 34.178951 & 109.028396 & 0 & 90 & E & & & & \\
\hline China, Tomb of Empress Xu (a) & 34.374648 & 108.68474 & -9.5 & 80.5 & $x$ & & & & \\
\hline China, Tomb of Empress Xu (b) & 34.12734 & 109.055786 & 0 & 90 & $E$ & & & & \\
\hline China, Tomb of Empress Zhang Yan & 34.423195 & 108.836961 & -15 & 75 & & & & & \\
\hline China, Tomb of Marquis Zhang Ao & 34.427745 & 108.851209 & -15 & 75 & & & & & \\
\hline China, Tomb of Princess Chengyang of Emperor Taizong & 34.6156 & 108.49314 & -6 & 84 & $x$ & & & & \\
\hline China, Tomb of Princess Xincheng of Emperor Taizong & 34.62365 & 108.49888 & -21 & 69 & & & & & \\
\hline China, Yarnaz Valley,Yarkhoto & 42.952022 & 89.061138 & -40 & 50 & $M$ & & & & \\
\hline India, Amritsar, Golden Temple & 31.619938 & 74.876511 & 33.2 & 123.2 & $M$ & & & & $\mathrm{E}$ \\
\hline India, Chidambaram, Chidambaram Nataraja & 11.399234 & 79.693715 & -1 & 89 & E & & & & \\
\hline India, Chitoor, Srikalahasti Temple & 13.749686 & 79.698308 & 0 & 90 & $\mathrm{E}$ & & & & \\
\hline India, Kanchipura, Ekambareswarar Temple & 12.847302 & 79.699525 & 18.3 & 108.3 & $\mathrm{~m}$ & & & & \\
\hline India, Khadirbet, Dholavira & 23.88690735 & 70.21377639 & -5 & 85 & & & & & \\
\hline India, Madhya Pradesh, Sas Bahu Temple & 16.018856 & 75.881959 & -4 & 86 & & & Z & & \\
\hline India, Madhya Pradesh, Tigawa Temple & 23.690196 & 80.066918 & -10 & 80 & & & E & & \\
\hline India, Mahabalipuram, Shore Temple & 12.616492 & 80.199267 & 13 & 103 & $z$ & s & & & \\
\hline India, Rameshwar Mandir & 16.21768 & 73.462012 & -14 & 76 & & $E$ & & & \\
\hline India, Shri Martand Sun Temple & 33.745588 & 75.220286 & -13.9 & 76.1 & & $E$ & & & \\
\hline India, Sigiriya & 7.957173 & 80.760031 & 8.3 & 98.3 & 2 & & & & $S$ \\
\hline India, Tamil Nadu & & & & & $\mathrm{E}, \mathrm{S}, \mathrm{Z}$ & & & & \\
\hline India, Thanjavur, Brihadisvara Temple & 10.782614 & 79.131735 & -20.5 & 69.5 & st, $m$ & & & & \\
\hline India, Tiruvannamalai, Annamalaiyar Temple & 12.231884 & 79.06679 & 11.4 & 101.4 & 2 & & & & \\
\hline India, Udaipur Rajasthan, Sas Bahu Temple & 24.735191 & 73.716283 & -16 & 74 & & $E$ & & & \\
\hline India, Venkateswara Temple & 13.68325 & 79.347195 & -7 & 83 & & & $\mathrm{E}$ & & \\
\hline Indonesia, Gunung Padang & -6.994518 & 107.056383 & -20 & 70 & & & & E & \\
\hline Inner Mongolia, Xanadu & 42.356388 & 116.184304 & 0 & 90 & E & & & & \\
\hline Japan, Osaka Castle & 34.687298 & 135.525826 & 5.7 & 95.7 & & & $E$ & & \\
\hline Maldives, Thinadhoo & 0.530107 & 72.99717 & 43 & 133 & D & & & & \\
\hline Pakistan, Harappa & 30.628104 & 72.863909 & 0 & 90 & $E$ & & & & \\
\hline Russia Por-Bazhyn & 50.615271 & 97.384872 & 9.5 & 99.5 & & & & s & \\
\hline Thailand Angkor Wat & 13.412469 & 103.866986 & 0 & 90 & $\mathrm{E}$ & & & & \\
\hline Thailand, Ayutthaya, Wat Phra Mahathat & 14.356943 & 100.567509 & -5.3 & 84.7 & $x$ & & & & \\
\hline Thailand, Kao Klang Nai, Sri Thep & 15.465521 & 101.144681 & 9.5 & 99.5 & & & Z & & \\
\hline Thailand, Prasat Hin Phimai & 15.22093 & 102.493861 & -22 & 68 & & & & $E$ & \\
\hline Thailand, Prasat Mueang Tam & 14.496089 & 102.982608 & -11 & 79 & & & & $z$ & \\
\hline Thailand, Prasat Phanom Rung & 14.532044 & 102.940223 & -5.5 & 84.5 & $x$ & & & & \\
\hline Thailand, Prasat Si Khoraphum & 14.944574 & 103.798352 & 0 & 90 & E & & & & \\
\hline Thailand, Wat Phra Sri Rattana Mahathat & 14.798673 & 100.613862 & 0 & 90 & $E$ & & & & \\
\hline
\end{tabular}

$\mathrm{D}=$ "sacred directions". $\mathrm{E}=$ cardinal directions, i.e. geographic poles and equinoxes. $\mathrm{M}, \mathrm{m}=$ major and minor lunar standstills. $\mathrm{S}=$ solstices. $\mathrm{st}=$ stellar alignments. $\mathrm{X}=$ magnetic pole at the time of construction. $Z$ = zenith passage. If no alignment is given, the reason is unknown. In some cases, there may be more than one explanation for an alignment. 


\section{TABLE 4}

\section{Alignments of Sites in Europe}

\begin{tabular}{|c|c|c|c|c|c|c|c|c|c|}
\hline Name & Latitude & Longitude & North & East & Current & Hudson Bay & Greenland & Norway Sea & Bering Sea \\
\hline Bosnia, Pyramid of the Sun & 43.977259 & 18.176514 & 8.4 & 98.4 & & & & & $\mathrm{E}$ \\
\hline Greece, Athens, The Parthenon & 37.971517 & 23.72659 & -13.5 & 76.5 & & & E & & \\
\hline Greece, Delphi Amphitheater & 38.482477 & 22.500577 & -38.2 & 51.8 & D & $\mathrm{E}$ & & & \\
\hline Greece, Knossos & 35.297863 & 25.163092 & 11.8 & 101.8 & & & $\mathrm{~m}$ & & $\mathrm{E}$ \\
\hline Greece, Mycenae, Lion Gate & 37.73075184 & 22.7564996 & -40 & 50 & & E & & & \\
\hline Greece, Mycenae, Tomb of Agamemnon & 37.726725 & 22.754367 & 10.5 & 100.5 & & & $\mathrm{~m}$ & & $\mathrm{E}$ \\
\hline Greece, The Temple of Artemis & 37.949611 & 27.363921 & 21 & 111 & & & & & \\
\hline Italy, Rome, Circus Maximus & 41.885944 & 12.485215 & 36.7 & 126.7 & $\mathrm{M}$ & & & & \\
\hline Italy, Rome, Palantine Hill & 41.889209 & 12.487459 & 36.7 & 126.7 & M & & & & \\
\hline Italy, Sardinia, Monte d'Accoddi & 40.79075445 & 8.448907568 & 9.1 & 99.1 & & & & $\mathrm{M}$ & $Z$ \\
\hline Malta, Gozo, Ġgantija Temple & 36.04726 & 14.269015 & 37 & 127 & $M$ & & & & \\
\hline Spain, Mosque-Cathedral of Cordoba & 37.878906 & -4.779387 & -30.4 & 59.6 & $\mathrm{~S}$ & & & & \\
\hline Spain, Naveta d'Es Tudons & 40.00307541 & 3.891652768 & -19.2 & 70.8 & $\mathrm{~m}$ & & & & \\
\hline Turkey, Hagia Sophia & 41.01314018 & 28.98318202 & 34.3 & 124.3 & $S$ & & & & \\
\hline Turkey, Hattusa & 40.01994347 & 34.61545489 & 38 & 128 & $M$ & $S$ & & $\mathrm{~m}$ & $\mathrm{~m}$ \\
\hline UK, Calanais Standing Stones & 58.197566 & -6.745127 & & & & & & & \\
\hline UK, Glastonbury Tor & 51.144444 & -2.698611 & -26.5 & 63.5 & $\mathrm{~m}$ & & & & \\
\hline UK, Stonehenge & 51.178868 & -1.826163 & & & $\mathrm{~S}, \mathrm{M}, \mathrm{m}$ & & & & \\
\hline
\end{tabular}

$\mathrm{D}=$ "sacred directions". $\mathrm{E}=$ cardinal directions, i.e. geographic poles and equinoxes. $\mathrm{M}, \mathrm{m}$ = major and minor lunar standstills. $S=$ solstices. If no alignment is given, the reason is unknown. In some cases, there may be more than one explanation for an alignment.

TABLE 5

\section{Alignments of Sites in North America}

\begin{tabular}{|c|c|c|c|c|c|c|c|c|c|}
\hline Name & Latitude & Longitude & North & East & Current & Hudson Bay & Greenland & Norway Sea & Bering Sea \\
\hline Canada, AB, Badlands Guardian & 50.01037 & -110.113133 & & & \begin{tabular}{|l|l|}
$E$ & \\
\end{tabular} & & & & \\
\hline US, California, Blythe Intaglios, B1 & 33.800585 & -114.532055 & 0 & 90 & E & & & & \\
\hline US, California,Blythe Intaglios, B3 & 33.800402 & -114.538078 & 29 & 119 & & $\mathrm{E}$ & & & \\
\hline US, Georgia, Ocmulgee National Monument & 32.838868 & -83.606114 & 34 & 124 & $\mathrm{M}$ & & & & \\
\hline US, New Mexico, Chaco Canyon, Pueblo del Arroyo & 36.060854 & -107.9663 & 24 & 114 & $\mathrm{M}$ & & & & \\
\hline US, Ohio, Great Serpent Mound & 39.02642 & -83.431091 & 27.7 & 117.7 & $S$ & & & & \\
\hline US. Illinois, Cahokia, Monks Mound & 38.660158 & -90.062466 & & & S,M & & & & \\
\hline
\end{tabular}

$E=$ cardinal directions, i.e. geographic poles and equinoxes. $M=$ major lunar standstills. $\mathrm{S}=$ solstices. In some cases, there may be more than one explanation for an alignment.

TABLE 6

Alignments of Sites in the Pacific Ocean

\begin{tabular}{|c|c|c|c|c|c|c|c|c|c|}
\hline Name & Latitude & Longitude & North & East & Current & Hudson Bay & Greenland & Norway Sea & Bering Sea \\
\hline Chile,Easter Island, Ahu Akivi & -27.115014 & -109.395043 & -2.7 & 87.3 & $\mathrm{E}$ & & & & \\
\hline Chile,Easter Island, Ahu Nau Nau & -27.074425 & -109.322455 & -19.6 & 70.4 & $\mathrm{~m}$ & & & & \\
\hline Chile,Easter Island, Ahu Tahai & -27.140076 & -109.427314 & 8.3 & 98.3 & & & $\mathrm{E}$ & & \\
\hline Chile,Easter Island, Ahu Tongariki & -27.125774 & -109.276933 & 30 & 120 & S & & & & \\
\hline Chile,Easter Island, Ahu Vinapu & -27.174098 & -109.405737 & 8.1 & 98.1 & & & $\mathrm{E}$ & & \\
\hline Micronesia, Nan Madol & 6.844537 & 158.335795 & -33 & 57 & $M$ & & $\mathrm{E}$ & & \\
\hline Micronesia, Nan Madol, Temple of Nan Dawas & 6.844537 & 158.335795 & 7 & 97 & $z$ & & $\mathrm{E}$ & & \\
\hline Samoa, Pulemelei Mound & -13.735237 & -172.324399 & -7.3 & 82.7 & & & & & \\
\hline Tonga, Ha'amonga 'a Maui Trilithon & -21.136606 & -175.048087 & 32.7 & 122.7 & & $\mathrm{E}$ & & & \\
\hline
\end{tabular}

$\mathrm{E}=$ cardinal directions, i.e. geographic poles and equinoxes. $\mathrm{M}, \mathrm{m}=$ major and minor lunar standstills. $S=$ solstices. $Z$ = zenith passage. If no alignment is given, the reason is unknown. In some cases, there may be more than one explanation for an alignment. 


\section{TABLE 7}

\section{Alignments of Sites in the Middle East}

\begin{tabular}{|c|c|c|c|c|c|c|c|c|c|}
\hline Name & Latitude & Longitude & North & East & Current & Hudson Bay & Greenland & Norway Sea & Bering Sea \\
\hline Iran, Chogha Zanbil & 32.00899687 & 48.5215934 & -43.5 & 46.5 & & $\mathrm{~m}$ & $\mathrm{M}$ & $\mathrm{m}$ & $\mathrm{S}$ \\
\hline Iraq, Dur-Kurigalzu & 33.35367069 & 44.20216381 & -39.6 & 50.4 & & & & S & $M$ \\
\hline Iraq, Tower of Babel & 32.536284 & 44.420803 & -11.3 & 78.7 & & & $\mathrm{E}$ & & \\
\hline Iraq, Ziggurat of Ur & 30.962711 & 46.103126 & -33.3 & 56.7 & $M$ & & & & \\
\hline Jerusalem, Dome of the Rock & 31.778087 & 35.235306 & -7.3 & 82.7 & $D$ & & & & \\
\hline Jerusalem, Western Wall & 31.776657 & 35.23447 & -12.1 & 77.9 & & & E & & \\
\hline Jordan, Petra, Temple of the Winged Lions & 30.330297 & 35.442554 & 17.5 & 107.5 & & & & & $\mathrm{E}$ \\
\hline Jordan, Qasr II-Abd, Irak Al-Amir & 31.912785 & 35.751941 & -15 & 75 & & & & $\mathrm{E}$ & \\
\hline Jordan, Umayyad Mosque in Amman & 33.51159288 & 36.3066567 & -6.4 & 83.6 & & & & & 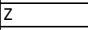 \\
\hline Lebanon, Baalbek, Temple of Jupiter & 34.006694 & 36.203826 & -12.2 & 77.8 & & & $\mathrm{E}$ & & \\
\hline Saudi Arabia, Mecca, Kaaba & 21.42251 & 39.826174 & -34.9 & 55.1 & $\mathrm{M}$ & & & & \\
\hline Turkey, Harran & 36.865021 & 39.031565 & 9.6 & 99.6 & & & $\mathrm{~m}$ & & \\
\hline Yemen, Great Mosque of Sana'a & 15.353123 & 44.214876 & -25 & 65 & $\mathrm{M}$ & & & & \\
\hline
\end{tabular}

$\mathrm{D}=$ "sacred directions." $\mathrm{E}=$ cardinal directions, i.e. geographic poles and equinoxes. $\mathrm{M}, \mathrm{m}=$ major and minor lunar standstills. $\mathrm{S}=$ solstices. $\mathrm{Z}=$ zenith passage. In some cases, there may be more than one explanation for an alignment.

\section{TABLE 8}

\section{Alignments of Sites in South America}

\begin{tabular}{|c|c|c|c|c|c|c|c|c|c|}
\hline Name & Latitude & Longitude & North & East & Current & Hudson Bay & Greenland & Norway Sea & Bering Sea \\
\hline Bolivia, Chincana Labyrinth & -15.990127 & -69.202952 & 44 & 134 & D & & & $\mathrm{S}$ & \\
\hline Bolivia, Puma Punku & -16.56172 & -68.680046 & 2 & 92 & & & $\mathrm{E}$ & & \\
\hline Bolivia, Quenuani & -16.259407 & -69.17127 & -20 & 70 & S & & S & & \\
\hline Bolivia, Tiwanaku & -16.554933 & -68.673487 & 2 & 92 & & & $\mathrm{E}$ & & \\
\hline Peru, Caral-Supe & -10.893458 & -77.52054 & 19.5 & 109.5 & $S$ & & & & \\
\hline Peru, Caral-Supe, Huanca Pyramid & -10.893458 & -77.52054 & 19.5 & 109.5 & & & & E & \\
\hline Peru, Chan Chan & -8.103554 & -79.07076 & 19.5 & 109.5 & $\mathrm{~m}$ & & & $\mathrm{E}$ & \\
\hline Peru, Chavin & -9.594527 & -77.177002 & 14.7 & 104.7 & $\mathrm{D}$ & & & & \\
\hline Peru, Cuzco & -13.518587 & -71.975952 & & & & & & & E \\
\hline Peru, Huanuco Pampa & -9.875388 & -76.816395 & 0 & 90 & $\mathrm{E}$ & & & & \\
\hline Peru, Huayna Picchu, Temple of the Moon & -13.151931 & -72.546507 & & & M & & & & \\
\hline Peru, La Centinela & -13.45007514 & -76.17223285 & 5.6 & 95.6 & & & & $z$ & \\
\hline Peru, Machu Picchu, Temple of the Three Windows & -13.163592 & -72.545414 & -34.7 & 55.3 & & & & & $\mathrm{E}$ \\
\hline Peru, Machu Picchu, Terraces & -13.164219 & -72.544831 & -25 & 65 & & s & & & \\
\hline Peru, Marcahuasi, Face & -11.77567 & -76.581853 & 43 & 133 & D & & & $S$ & \\
\hline Peru, Nazca Lines & -14.712825 & -75.17485 & 19.3 & 109.3 & $\mathrm{D}$ & & & E & E \\
\hline Peru, Ollantaytambo, Temple of the Sun & -13.257536 & -72.267129 & -35 & 55 & & & & & $\mathrm{E}$ \\
\hline Peru, Sacsahuaman & -13.50933 & -71.980916 & & & $\mathrm{D}$ & & & & \\
\hline Peru, Sechin Bajo & -9.4648088 & -78.26525923 & -25.5 & 64.5 & $S$ & & & & \\
\hline Peru, Warawtampu & -10.46549 & -76.536647 & -24.2 & 65.8 & D & & & & \\
\hline Peru. Chotuna & -6.720363 & -79.952796 & 0 & 90 & $\mathrm{E}$ & & & & \\
\hline
\end{tabular}

$\mathrm{D}=$ "sacred directions." $\mathrm{E}=$ cardinal directions, i.e. geographic poles and equinoxes. $\mathrm{M}, \mathrm{m}=$ major and minor lunar standstills. $\mathrm{S}=$ solstices. $\mathrm{Z}=$ zenith passage. In some cases, there may be more than one explanation for an alignment.

Of the 95 unexplained sites identified in our initial study, the shifted pole model is able to explain all but 17 of the alignments. 62 sites face one of the previous pole locations, 21 to solstices, and 21 to lunar standstills that reference previous pole locations. In some cases a site had more than one alignment; e.g., Knossos appears to be both 
TABLE 9

Alignments of Sites in Mesoamerica

\begin{tabular}{|c|c|c|c|c|c|c|c|c|c|}
\hline Name & Latitude & Longitude & North & East & Current & Hudson Bay & Greenland & Norway Sea & Bering Sea \\
\hline Belize, Altun $\mathrm{Ha}$, Sun God Pyramid & 17.76395 & -88.347061 & 7.6 & 97.6 & & E & & & \\
\hline Belize, Xunantunich & 17.088922 & -89.141631 & -10.3 & 79.7 & D & & & & \\
\hline El Salvador, Tazumal & 13.979547 & -89.674131 & 18 & 108 & $\mathrm{~m}$ & & & & \\
\hline Guatemala, Mixco Viejo & 14.871668 & -90.664167 & 12.5 & 102.5 & $\mathrm{D}$ & E & & & \\
\hline Guatemala, Tikal & 17.222094 & -89.623614 & 8.6 & 98.6 & & $\mathrm{E}$ & & & \\
\hline Guatemala, Yaxchilan & 16.899655 & -90.967093 & 30.4 & 120.4 & $\mathrm{D}$ & & & & \\
\hline Honduras, Copan, Step Pyramids & 14.84 & -89.14 & & & $z$ & & & & \\
\hline \begin{tabular}{|l|} 
Mexico, Acatitlan \\
\end{tabular} & 19.55 & -99.17 & 20.3 & 110.3 & $\mathrm{Z}$ & & & $\mathrm{E}$ & \\
\hline Mexico, Alta Vista & 23.478544 & -103.945607 & & & & $\mathrm{~S}, \mathrm{M}, \mathrm{m}$ & & & \\
\hline Mexico, Bonampak & 16.704 & -91.065 & 38 & 128 & & & $M$ & & \\
\hline Mexico, Calakmul & 18.105392 & -89.810829 & 8.8 & 98.8 & & $\mathrm{E}$ & & & \\
\hline Mexico, Calixtlahuaca & 19.335038 & -99.69757 & -30 & 60 & $M$ & & & & \\
\hline Mexico, Chalcatzingo & 18.676715 & -98.770783 & 6.8 & 96.8 & & & $\mathrm{E}$ & & \\
\hline Mexico, Chichen Itza & 20.68 & -88.57 & 21 & 111 & $\mathrm{M}, \mathrm{Z}$ & & & $\mathrm{E}$ & \\
\hline Mexico, Chimalacatlan, C1 & 18.446236 & -99.105878 & -34.7 & 55.3 & $\mathrm{M}$ & & & & E \\
\hline Mexico, Chimalacatlan, C2 & 18.444804 & -99.104331 & 28.7 & 118.7 & $\mathrm{~m}$ & & & & $\mathrm{~S}$ \\
\hline Mexico, Cholula & 19.0583048 & -98.30190553 & 25 & 115 & S & & & & \\
\hline Mexico, Coba, Grand Pyramid & 20.492974 & -87.724195 & -39 & 51 & & $M$ & & & \\
\hline Mexico, Comalcalco & 18.27819958 & -93.20032665 & 24 & 114 & $S$ & & & & \\
\hline Mexico, Cuauhtinchan Archeological Site, Cuauhcalli & 18.9535 & -99.502888 & 15.4 & 105.4 & $\mathrm{~m}$ & & & & \\
\hline Mexico, Cuicuilco & 19.301021 & -99.183798 & & & & & & & \\
\hline Mexico, El Cerrito Archaological Zone & 20.551376 & -100.444027 & 7.4 & 97.4 & & & $\mathrm{E}$ & & \\
\hline Mexico, El Tajin, Pyramid of the Niches & 20.448058 & -97.378242 & 14.5 & 104.5 & & $\mathrm{E}$ & & & \\
\hline Mexico, El Tajin, Southern Ballcourt & 20.448058 & -97.378242 & 0 & 90 & $\mathrm{E}$ & & & & \\
\hline Mexico, El Tajin, Tajin Chico & 20.448058 & -97.378242 & 40 & 130 & & & & & \\
\hline Mexico, El Tepozteco & 19.00078611 & -99.1015579 & 26 & 116 & $\mathrm{~m}$ & & & & \\
\hline Mexico, La Venta & 18.103191 & -94.040946 & -12.2 & 77.8 & D & & & & \\
\hline Mexico, Mayapan & 20.629823 & -89.46059 & & & & & & & \\
\hline Mexico, Mitla & 16.92704923 & -96.35934812 & 12 & 102 & & $\mathrm{E}$ & & & \\
\hline Mexico, Monte Alban & 17.042122 & -96.768184 & 6.45 & 96.45 & & & $\mathrm{E}$ & & \\
\hline Mexico, Monte Alban, Building J & 17.042122 & -96.768184 & -43 & 47 & st & & & & \\
\hline Mexico, Palenque, North Group & 17.483978 & -92.04632 & 10.1 & 100.1 & & $\mathrm{E}$ & & & \\
\hline Mexico, Palenque, Temple of the Inscriptions & 17.48 & -92.05 & 20.6 & 110.6 & & & & $\mathrm{E}$ & \\
\hline Mexico, Tenango & 19.108425 & -99.597693 & 14 & 104 & & $\mathrm{E}$ & & & \\
\hline Mexico, Tenochtitlan & 19.435 & -99.131389 & 7 & 97 & & & $\mathrm{E}$ & & \\
\hline Mexico, Teotihuacan & 19.6925 & -98.843889 & 15.6 & 105.6 & st & $\mathrm{E}$ & & & \\
\hline Mexico, Tiatelolco & 19.450994 & -99.13751 & 8.5 & 98.5 & & & & & \\
\hline Mexico, Tula & 20.064451 & -99.3405 & 15.47 & 105.47 & $\mathrm{~m}$ & & & & \\
\hline Mexico, Tulum & 20.21 & -87.43 & 22.3 & 112.3 & & & & $\mathrm{E}$ & \\
\hline Mexico, Uxmal, Palace of the Governors & 20.359444 & -89.771389 & 30 & 120 & $\mathrm{~S}$ & & & & \\
\hline Mexico, Uxmal, Pyramid of the Magician & 20.359444 & -89.771389 & 9.2 & 99.2 & & $\mathrm{E}$ & & & \\
\hline Mexico, Uxmal, Templo Mayor & 20.359444 & -89.771389 & 19.6 & 109.6 & $\mathrm{Z}$ & & & $\mathrm{E}$ & \\
\hline Mexico, Xochicalco, Grand Pyramid & 18.803889 & -99.295917 & 0 & 90 & $\mathrm{E}$ & & & & \\
\hline Mexico, Xochicalco, Temple of Quetzalcoatl & 18.803889 & -99.295917 & 15.4 & 105.4 & & $\mathrm{E}$ & & & \\
\hline
\end{tabular}

$\mathrm{D}=$ "sacred directions." $\mathrm{E}=$ cardinal directions, i.e. geographic poles and equinoxes. $\mathrm{M}, \mathrm{m}=$ major and minor lunar standstills. $\mathrm{S}=$ solstices. $\mathrm{st}=$ stellar alignments. $\mathrm{Z}=$ zenith passage. If no alignment is given, the reason is unknown. In some cases, there may be more than one explanation for an alignment.

cardinally aligned to the Bering Sea pole and aligned to minor lunar standstills relative to the Greenland pole.

Figure 2 shows 4 of the 18 sites found that face the Hudson Bay pole. All of Teotihuacan is aligned to the Hudson Bay pole, as are structures in Tikal's North Acropolis. The Sri Martand Sun Temple in India does not currently face the sun, but if it were originally built when the North Pole was in Hudson Bay it would have been aligned as many sun temples are to the cardinal directions at that time. An extended area 


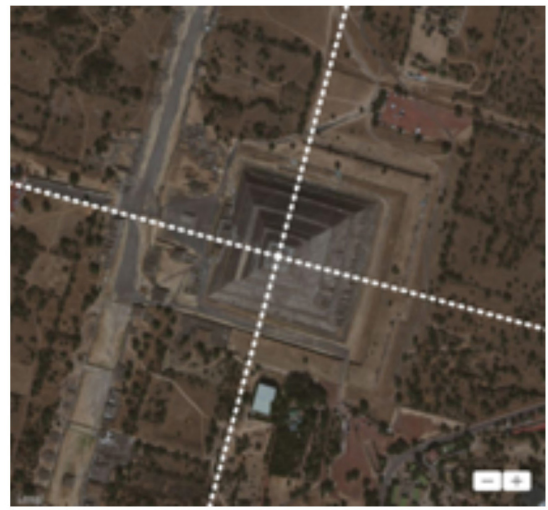

A) Teotihuacan, Mexico

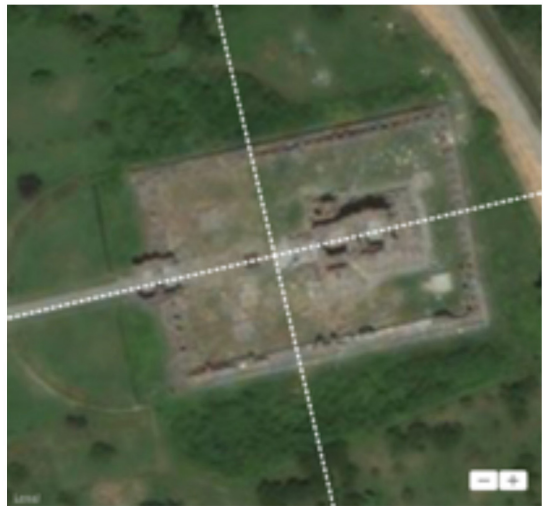

C) Sri Martand Sun Temple, India

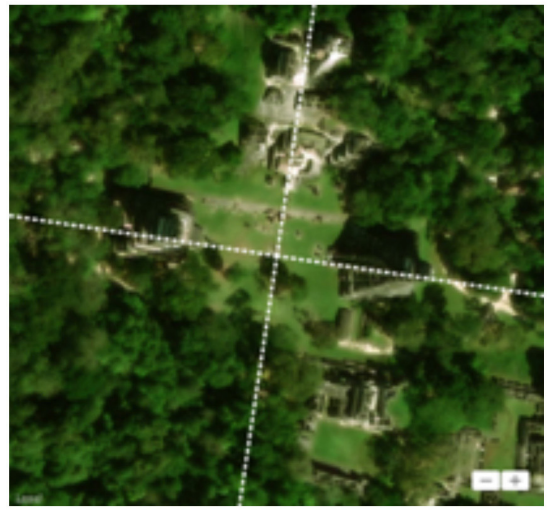

B) Tikal, Guatemala

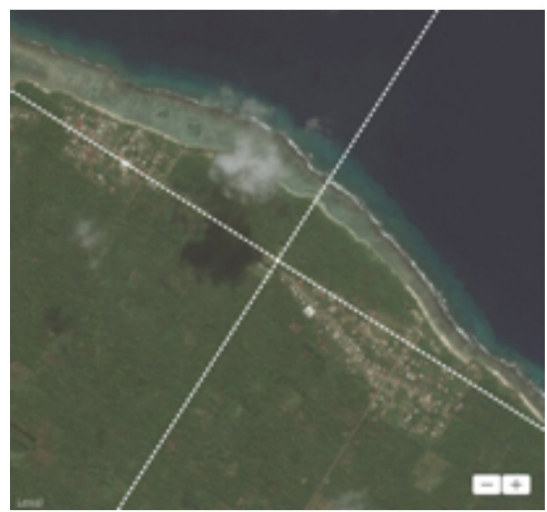

D) Ha'amonga ‘a Maui

Figure 2. Examples of sites aligned to the Hudson Bay pole. Photo credit: Apple Maps.

on the island of Tonga surrounding the Ha'amonga 'a Maui Trilithon also is oriented in the direction of the Hudson Bay pole.

Twenty sites were found that face the Greenland pole. Four of the sites are shown in Figure 3. The Temple of Jupiter at Baalbek was built by the Romans over an earlier pre-Roman structure (Lohmann, 2010). Similarly, the Parthenon atop the Acropolis in Athens was built over an earlier Parthenon (Beard, 2010). Hannah (2013) reviews Dinsmoor's analysis of the Parthenon and concludes that on August 31, $488 \mathrm{BCE}$, Athena's "birthday," the sun would have risen north of east along the main axis of the temple. But how do we know when Athena, a goddess, 


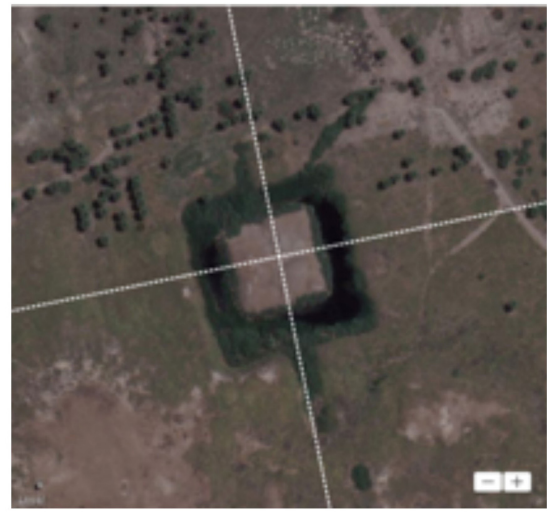

A) Tower of Babel, Babylon

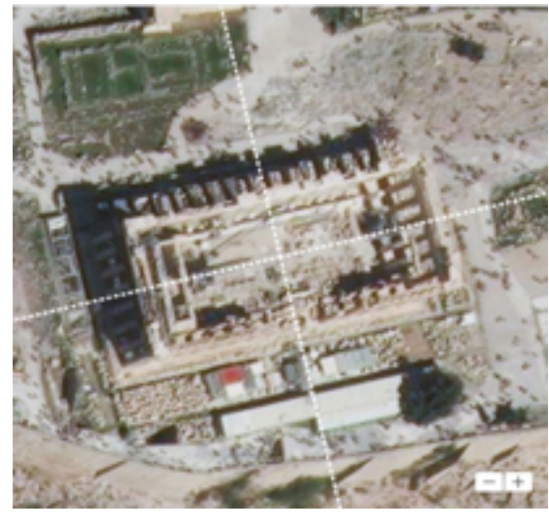

C) The Parthenon, Athens

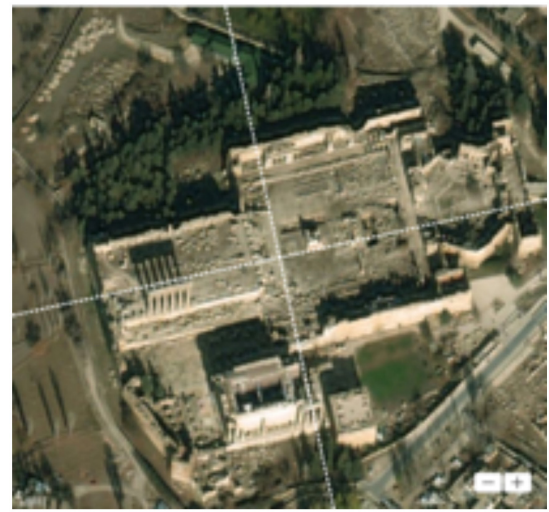

B) Temple of Jupiter, Baalbek, Lebanon

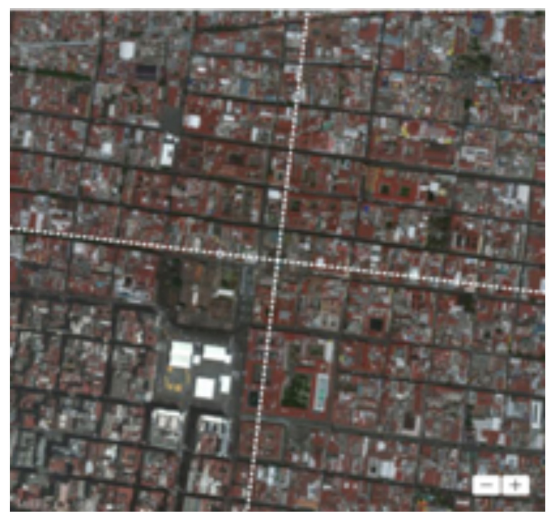

D) Tenochtitlan, Mexico City

Figure 3. Examples of sites aligned to the Greenland pole. Photo credit: Apple Maps.

the daughter of Zeus, was born? Was the Parthenon aligned with the sunrise on Athena's birthday, or was the date of Athena's birthday established based on the preexisting orientation of the Parthenon? And was this orientation, along with other structures on the Acropolis, originally directed toward an ancient pole in Greenland?

Chichen Itza and El Tepozteco in Mexico, Caral Supe in Peru, and the Brihadisvara Temple in India are 4 of the 12 sites found that face the Norwegian Sea pole (Figure 4). Southwest of the Temple of Quetzalcoatl 


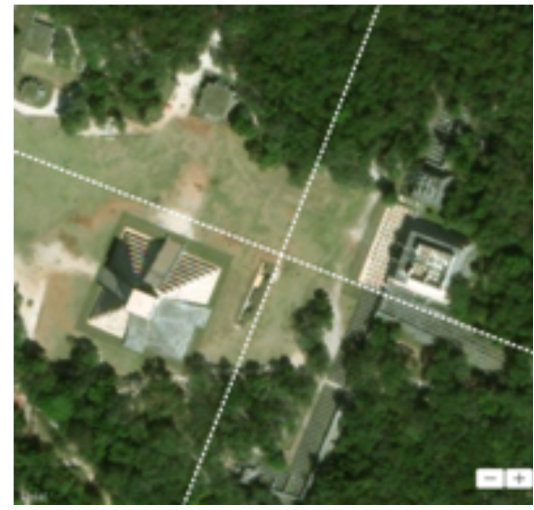

A) Chichen Itza, Mexico

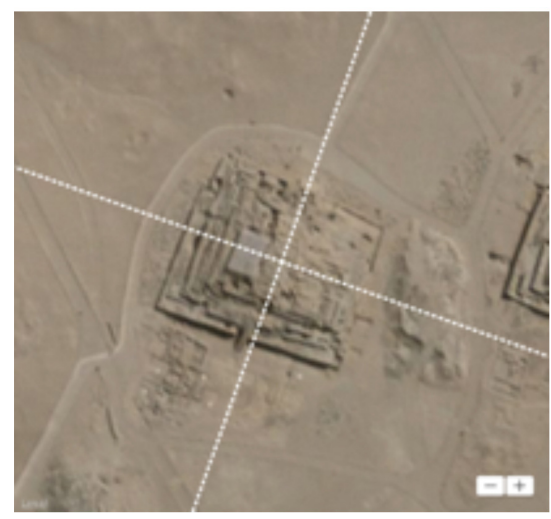

C) Caral-Supe, Peru

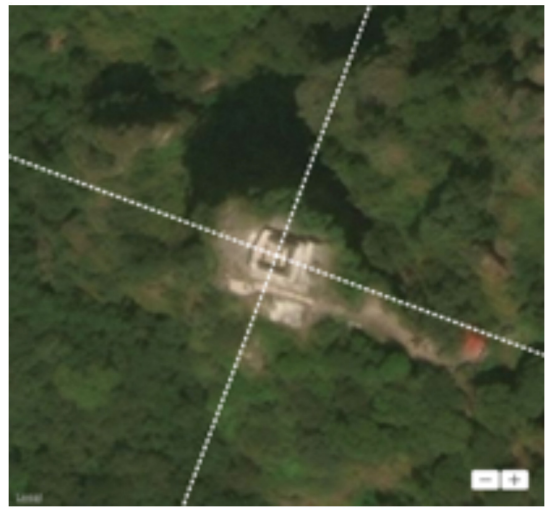

B) El Tepozteco, Mexico

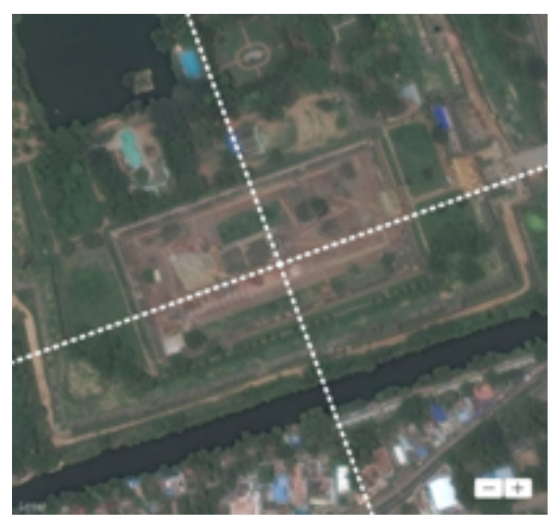

D) Brihadisvara Temple, India

Figure 4. Examples of sites aligned to the Norwegian Sea pole. Photo credit: Apple Maps.

at Chichen Itza, the Caracol is a dome-shaped structure thought to have been an observatory aligned to celestial events, including the summer and winter solstice sunrises and sunsets and the setting of the planet Venus. If this were its intended purpose, why are the Caracol, as well as the Temple of Quetzalcoatl and numerous other structures at Chichen Itza, all oriented in a decidedly non-solar direction, approximately $21.5^{\circ}$ east of north, in the direction of the Norway pole?

Figure 5 shows 4 of the 12 sites that have been found to be 


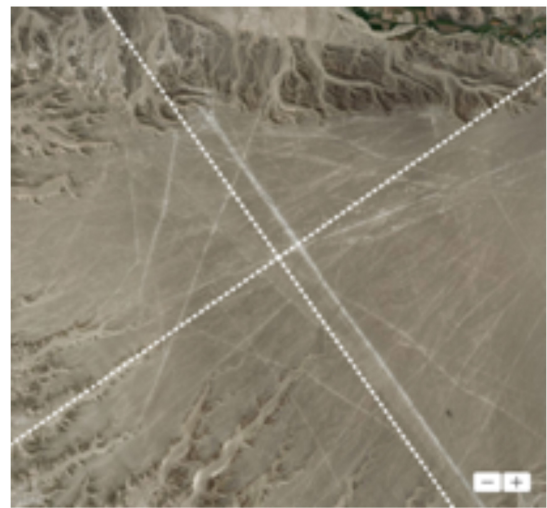

A) Nazca line, Peru

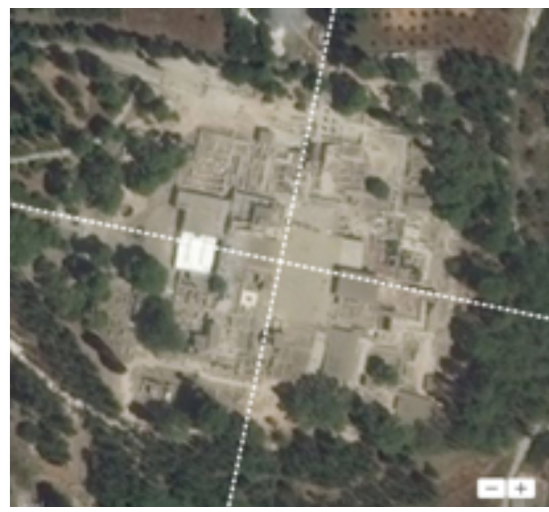

C) Knossos, Crete

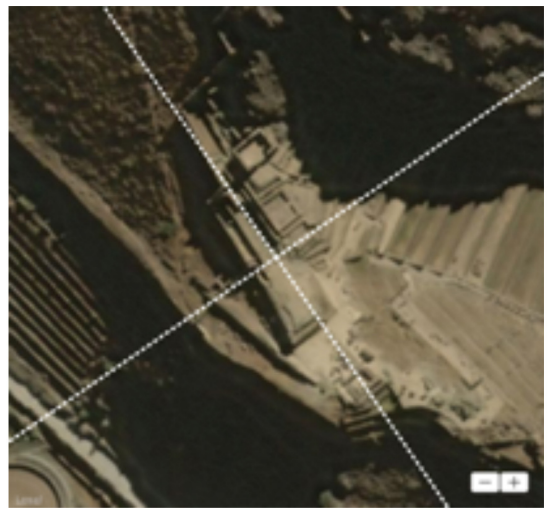

B) Temple of the Sun, Ollantaytambo, Peru

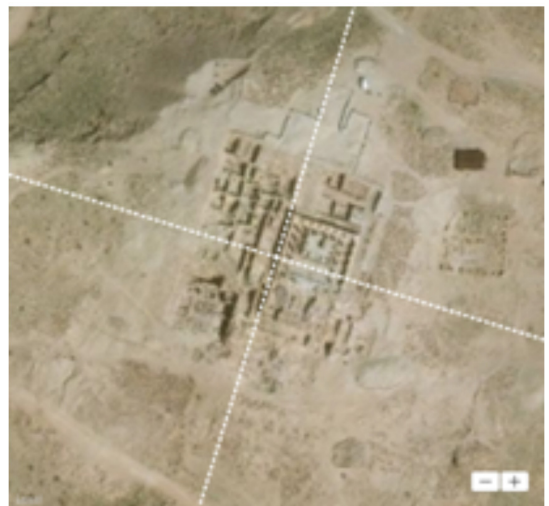

D) Temple of the Winged Lions, Petra, Jordan

Figure 5. Examples of sites aligned to the Bering Sea pole. Photo credit: Apple Maps.

aligned to the Bering Sea pole. One of the major Nazca lines is in the direction of the pole (another is in line with the Norwegian Sea pole). The Temple of the Sun at Ollantaytambo in Peru and the Temple of the Three Windows at Machu Picchu are aligned to the Bering Sea pole as are Knossos in Crete and the Temple of the Winged Lions in Petra, Jordan. The direction of the Bering Sea pole is also closely aligned with a pattern of lines called ceques emanating out from the City of Cuzco.

In addition to sites aligned to the cardinal directions, the shifted pole alignment model accounts for 42 previously unexplained sites that could once have been aligned to solstices and to lunar standstills. Four 


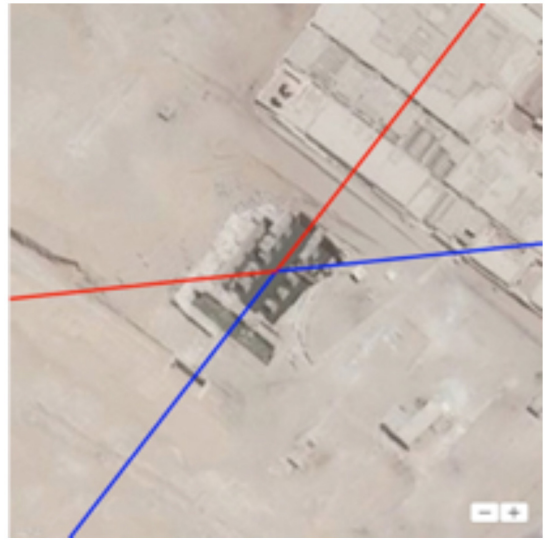

A) Osireon, Abydos, Egypt

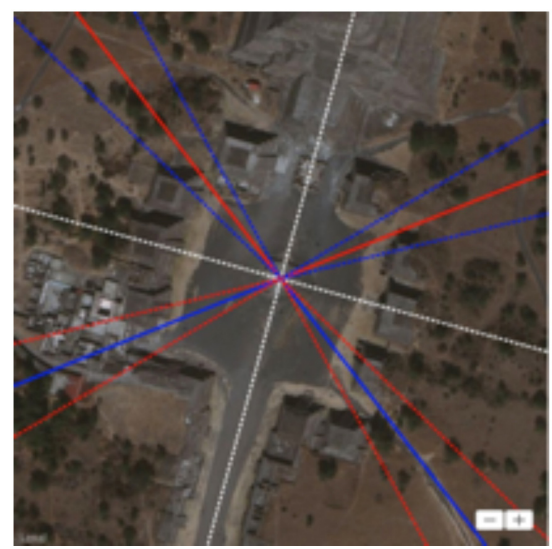

C) Plaza below Pyramid of the Moon, Teotihuacan, Mexico

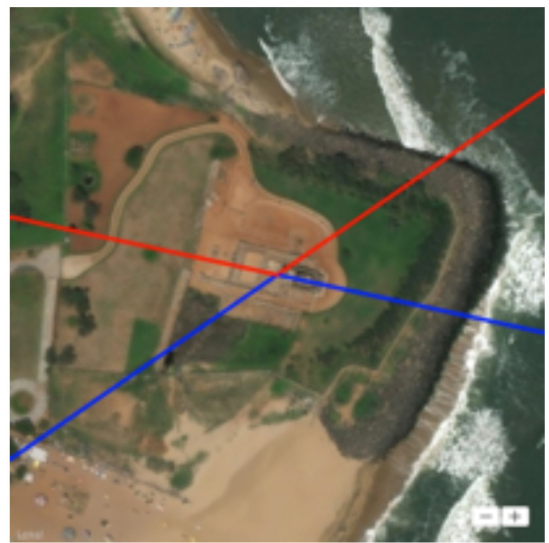

B) Shore Temple, Mahabalipuram, India

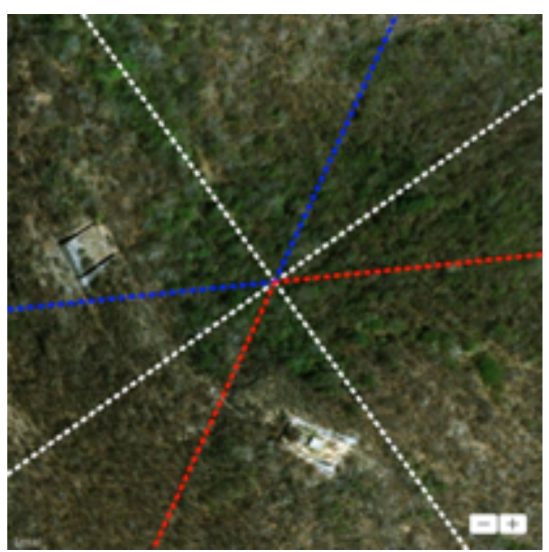

D) Two structures, Chimalacatlan, Mexico

Figure 6. Examples of other sites that reference previous pole locations. Pairs of solid lines are the summer and winter solstice alignments. Dotted lines are lunar standstill directions. A) is aligned to the summer solstice sunrise/winter solstice sunset relative to the Hudson Bay pole. B) is aligned to the winter solstice sunrise/summer solstice sunset relative to the Hudson Bay pole. C) shows solar and lunar alignments relative to the Hudson Bay pole. D) is aligned to cardinal directions and major lunar standstills relative to the Bering Sea pole. Photo credit: Apple Maps.

of the sites are shown Figure 6. The Osireon in Abydos is thought to have been an integral part of Seti l's funerary temple yet it was originally built at a considerably lower level than the foundations of the temple (Petrie \& Murray, 1903). It is not currently aligned in any direction 
of astronomical significance. According to our proposed model, the Osireon would be aligned to solstices if the North Pole were in Hudson Bay. This is also the case for the Shore Temple in Mahabalipuram, India. At Teotihuacan, the Pyramid of the Moon, the Pyramid of the Sun, the Temple of Quetzalcoatl, and the Avenue of the Dead all are aligned in the direction of the Hudson Bay pole. Numerous other structures in the plaza south of the Pyramid of the Moon that do not now reference any obvious astronomical events would have been aligned to solstices and lunar standstills at that time. Structures at Chimalacatlan in Mexico (Vigato, 2015) also appear to reference the Bering Sea pole.

Figure 7 plots the distribution of site alignments within the eight geographic regions versus pole location. Although the sample size is somewhat limited, certain patterns are evident. There are far more sites in Africa and Asia that are currently aligned to the cardinal directions than to any other direction at any other time. Over time, the number of sites appears to have increased in Mesoamerica and decreased in South America. Most of the sites in these regions were aligned to the cardinal directions. On the other hand, most sites in Europe and the Middle East were aligned to the moon. Where sites exist from the time of the Bering Sea pole to the present in six of the eight regions, there are no sites in the Pacific before the Greenland pole or in North America before the Hudson Bay pole.

\section{DISCUSSION}

Aveni (1980) states that modern cities tend to be built over the sites of earlier settlements, often preserving the original alignments for convenience of construction, and notes that the alignments of churches and planted fields in certain regions of Mexico follow the directions of alignments that had already been established in pre-Columbian times. Our hypothesis is that, over time, as certain sites fell into ruin, they were rebuilt and expanded, and new structures were added above and/ or around them consistent with the original site plan. What remains today thus indicates the original alignment of the site. In sites that contain both cardinally aligned and non-aligned structures there might not be obvious differences between the two if the older non-aligned structures were rebuilt or built over at the same time new cardinally aligned structures were added. Perhaps deeper excavations at these 

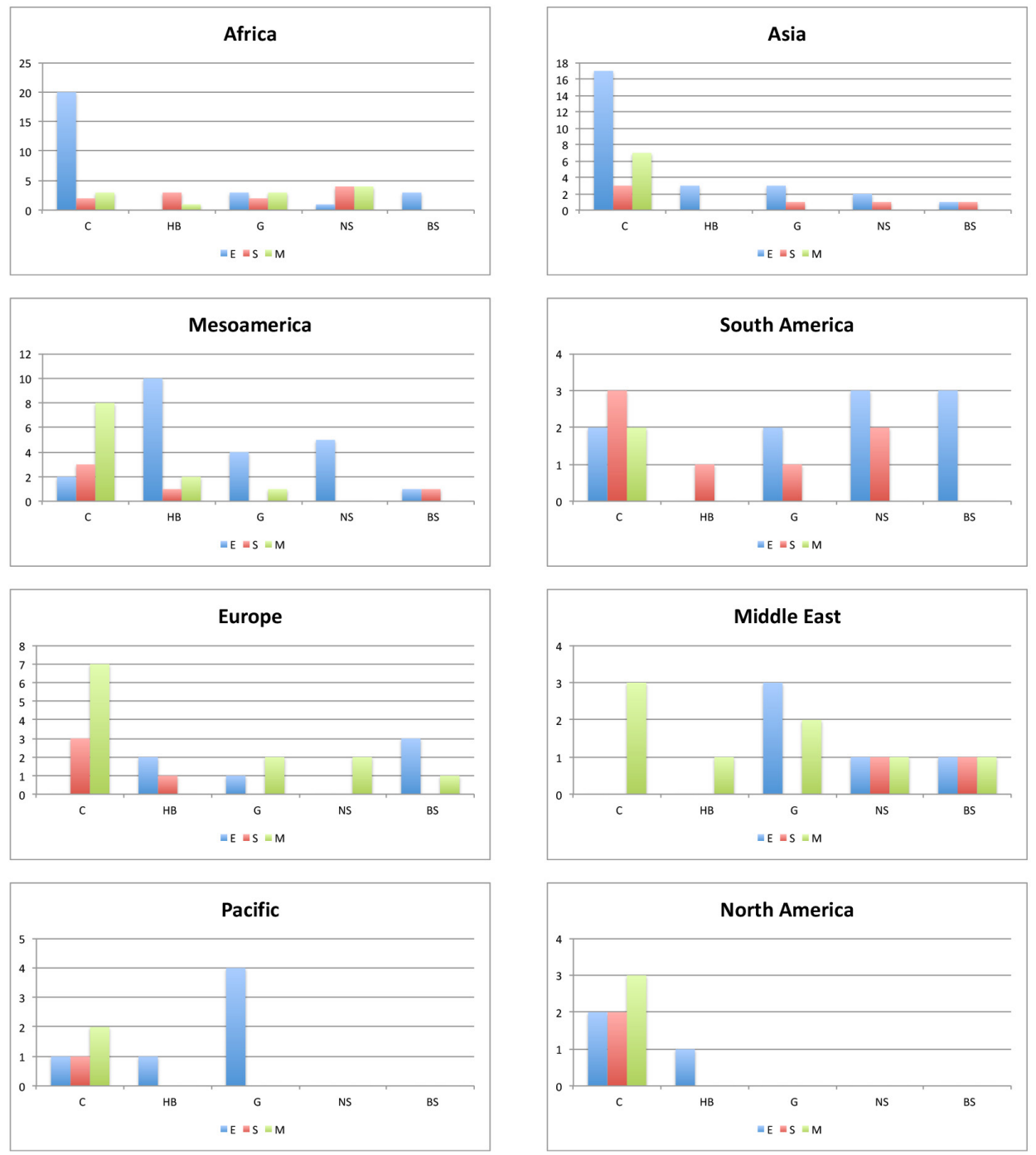

Figure 7. Distributions of alignments within all eight geographic regions. Each graph plots the number of equinox (E), solstice $(S)$, and lunar standstill (M) alignments relative to the current geographic pole (C), and the four hypothesized prior pole locations in Hudson Bay (HB), Greenland (G), the Norwegian Sea (NS), and the Bering Sea (BS).

sites would provide further evidence of the antiquity of the non-aligned structures.

The proposed shifted geographic pole alignment model explains 
many archeoastronomical enigmas including the distributions of temple and pyramid alignments in Mesoamerica and previously unexplained alignments of certain megalithic structures at Baalbek, Abydos, Machu Picchu, Ollantaytambo, and in other places. In analyzing the alignment of archaeological sites in Mexico, Aveni and Hartung conclude that an eastern skew was a standard architectural practice over a wide area in Mexico (Aveni, 2001). By accounting for the alignment of all but four of the Mesoamerican sites examined, the shifted pole model explains the reason for the skew.

The geographic pole shift hypothesis also provides a plausible explanation for the apparent lack of astronomical alignments of temples in Upper Egypt (Shaltout \& Belmonte, 2005) that is in stark contrast to the precise alignment of numerous pyramids in Lower Egypt to the cardinal directions. As shown in Figure 8, there are more structures in Lower Egypt that are aligned to the current geographic pole than in Upper Egypt. Conversely there are more structures in Upper Egypt that are aligned to previous geographic pole locations than in Lower Egypt. A geographic pole shift from Hudson Bay to the Arctic would have rotated this part of the world approximately $30^{\circ}$ (Figure 9) and displaced a considerable amount of water likely inundating low-lying areas along the Mediterranean Sea including Lower Egypt. A sudden shift of the crust would likely have triggered numerous earthquakes along fault lines. The temples in Upper Egypt lie in the Nile River valley far from large open bodies of water and several hundred miles west of the nearest tectonic plate. Perhaps by virtue of their more protected location, structures aligned to previous poles in Upper Egypt survived the crustal displacement and are therefore more numerous than those in Lower Egypt that were likely destroyed at the time.

That a model capable of explaining the alignment of so many archaeological sites that cannot otherwise be explained is itself predicted on Hapgood's unproven hypothesis, is problematic. It is possible that one day Hapgood's hypothesis may be verified by new discoveries in the earth sciences much like Wegner's theory of continental drift was. Although the idea that pole shifts were caused by an asymmetrical buildup of polar ice was rejected at the time, Hapgood's collaborator, J. $\mathrm{H}$. Campbell, developed a model that showed how materials rising out of / sinking into the lithosphere create imbalances in the mass distribution of the crust such that an area of increasing mass has the effect of 


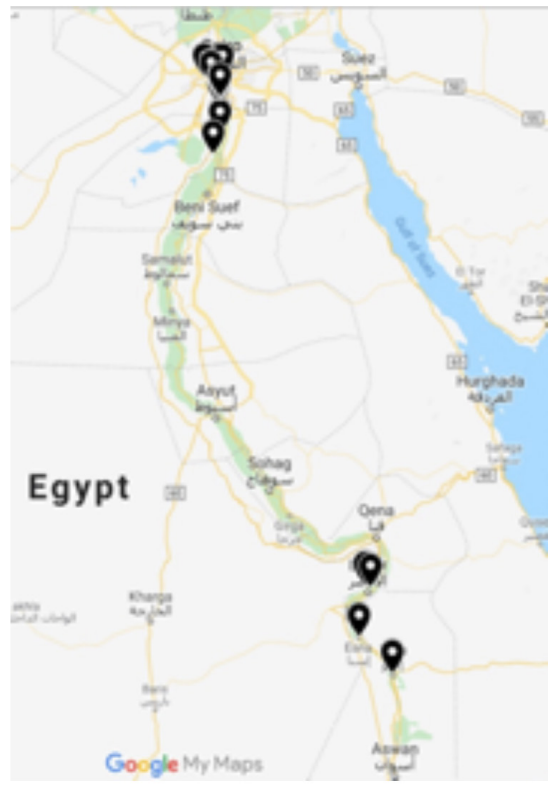

A) Alignments to current geographic pole

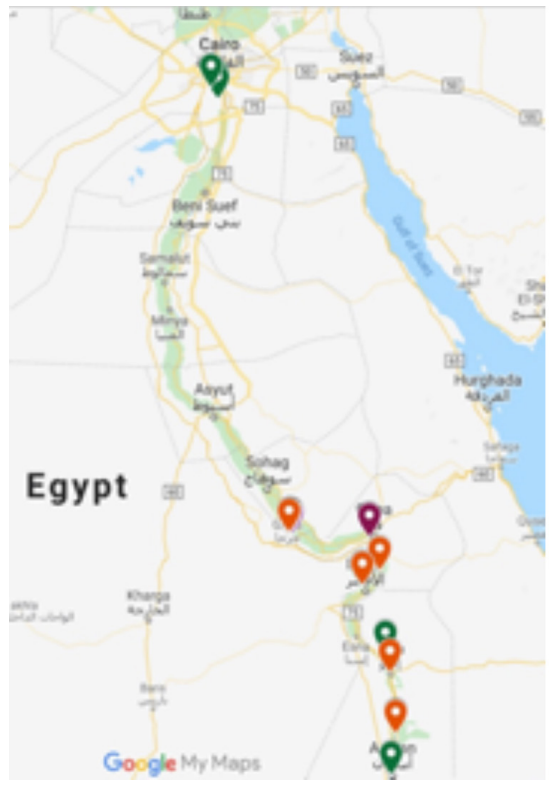

B) Alignments to previous geographic poles

Figure 8. Comparison of the number of sites in Lower Egypt (top of each map) and Upper Egypt (bottom of each map) aligned to the current geographic pole A) and previous hypothesized pole locations B).

A) There are 4 sites aligned to the current geographic pole in Upper Egypt and 20 sites in Lower Egypt.

B) There are 3 sites aligned to previous geographic poles in Lower Egypt and 13 sites in Upper Egypt. Photo credit: Google Maps.
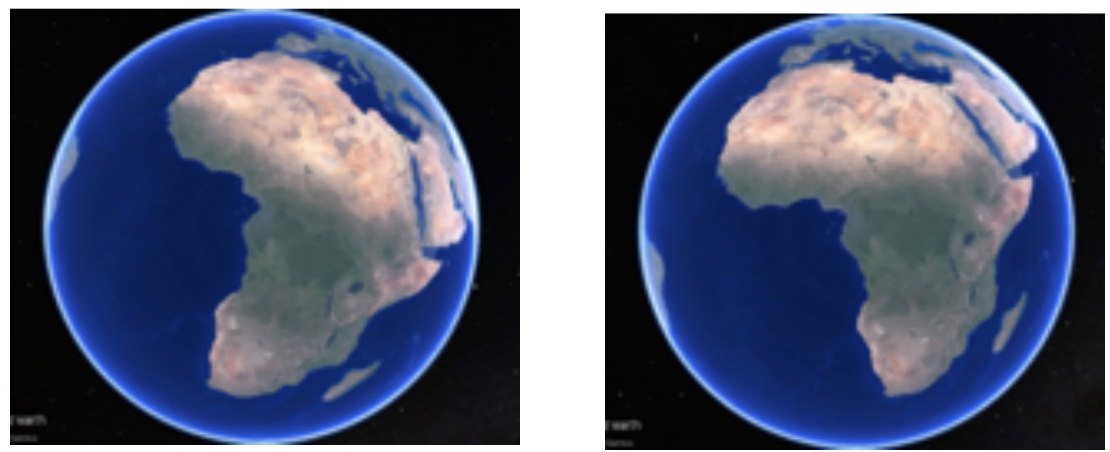

Figure 9. If the North Pole were in Hudson Bay (left), Europe and Africa would be rotated approximately $30^{\circ}$ clockwise relative to their current position (right). Photo credit: Google Earth. 
rotating the crust toward the equator and an area of decreasing mass has the opposite effect of rotating the crust toward the pole (Hapgood, 1958). The Tharsis formation on Mars is an example of how a large mass imbalance is thought to have shifted the Martian poles $20^{\circ}$ approximately 3.5 billion years ago (Bouley et al., 2016). Kirschvink et al. (1997) hypothesized that the movement of continental landmasses about a half billion years ago shifted Earth's North Pole by $90^{\circ}$.

As noted earlier, Chen at al. (2013) showed that small changes in the weight distribution of the crust caused by climate change induce small changes in the movement of the geographic pole. The current sizes of the Antarctic and Greenland ice sheets are approximately $1.3 \mathrm{X}$ $10^{19} \mathrm{~kg}$ and $2.7 \times 10^{18} \mathrm{~kg}$, respectively, which are two or more orders of magnitude smaller than Tharsis $\left(10^{21} \mathrm{~kg}\right.$ ). Twenty thousand years ago the Greenland Ice Sheet is estimated to have been almost ten times larger (Blue Marble, 2017) and could have been much thicker. When the mass of the Greenland Ice Sheet was comparable to that of Tharsis, large changes in it could have resulted in large changes in the movement of the Earth's geographic pole.

In his dismissal of theories of ancient civilizations, Brass (2002) states that there is no paleomagnetic evidence for Earth crustal displacements having occurred. As noted earlier, Kirschvink et al. (1997) concluded from paleomagnetic data collected in Australia and North America that a massive crustal shift did occur between 534 million and 505 million years ago. Paleomagnetic dating methods are intended to measure geological processes that occur over timescales of millions of years. Although it is beyond the scope of the present article to elaborate on this point, the absence of paleomagnetic evidence of Hapgood pole shifts may not be evidence of absence but could be due to the inability of paleomagnetic dating methods to temporally resolve and thus detect climate-induced events occurring over timescales that are two or more orders of magnitude faster than tectonic processes.

\section{REFERENCES}

Adhikari, S., \& Ivins, E. R. (2016). Climate-driven polar motion: 2003-2015. Science Advances, 2(4). Article e1501693. https://doi.org.10.1126/sciadv.1501693

Adhikari, S., Caron, L., Steinberger, B., Reager, J. T., Kjeldsen, K. K., Marzeion, B., Larour, E., \& Ivins, E. R. (2018). What drives 2oth century polar motion? Earth and Planetary Science Letters, 502, 126-132.

Aveni, A. (1980/2001). Skywatchers of ancient Mexico. University of Texas Press. pp. 236-238. 
Beard, M. (2010). The Parthenon. Harvard University Press.

Blue Marble. (2017). Blue Marble: Sea level, ice and vegetation changes—19,000 B.C.-10,000 A.D. https://sos.noaa.gov/datasets/blue-marble-sea-level-ice-andvegetation-changes-1900obc-10000ad/

Bouley, S., Baratoux, D., Matsuyama, I., Forget, F., Séjourné, A., Turbet, M., \& Costard, F. (2016, March). Late Tharsis formation and implications for early Mars. Nature, 531, 344-347.

Brass, M. (2002, July-August). Tracing Graham Hancock's shifting cataclysm. Skeptical Inquirer, 26(4), 46-49.

Carlotto, M. J. (2019, April 15-17). Archaeological dating using a data fusion approach. Paper presentation at Signal Processing, Sensor/Information Fusion, and Target Recognition XXVIII, Baltimore MD. SPIE: The International Society for Optics and Photonics. https://doi.org/10.1117/12.2520130

Carlotto, M. J. (2020). An analysis of the alignment of archaeological sites. Journal of Scientific Exploration, 34(1), 13-50.

Chen, J. L., Wilson, C. R., Ries, J. C., \& Tapley, B. D. (2013). Rapid ice melting drives Earth's pole to the east. Geophysical Research Letters, 40(11), 2625-2630. https://doi.org/10.1002/grl.50552

Daradich, A., Huybers, P., Mitrovica, J. X., Chan, N.-H., \& Austermann, J. (2017). The influence of true polar wander on glacial inception in North America. Earth and Planetary Science Letters, 461, 96-104. https://doi.org/10.1016/j.epsl.2016.12.036

Hannah, R. (2013). Greek temple orientation: The case of the older Parthenon in Athens. Nexus Network Journal, 15(3), 423-443. https://doi.org/10.1007/s00004-013-0169-1

Hapgood, C. H. (1958). Earth's shifting crust: A key to some basic problems of earth science (Foreword by Albert Einstein). Pantheon.

Holmes, A. (1944). Principles of physical geology. Thomas Nelson \& Sons.

Kious, W. J., \& Tilling, R. I. (1996). This dynamic Earth: The story of plate tectonics. U.S. Government Printing Office. https://pubs.usgs.gov/gip/dynamic/dynamic.html

Kirschvink, J. L., Ripperdan, R. L., \& Evans, D. A. (1997). Evidence for a largescale reorganization of Early Cambrian continental masses by inertial interchange true polar wander. Science, 277(5325), 541-545. https://doi. org/10.1126/science.277.5325.541

Lohmann, D. (2010). Giant strides towards monumentality-The architecture of the Jupiter Sanctuary in Baalbek / Heliopolis. Bollettino di Archeologia. http://www.daniellohmann.net/dox/lohmann_aiac2008.pdf

Milanković, M. (1932). [Numerical trajectory of secular changes of pole's rotation.] Academy of National Sciences Belgrade. http://elibrary.matf.bg.ac.rs/ bitstream/handle/123456789/3675/mm35F.pdf?sequence $=1$

Muller, R. A., \& MacDonald, G. J. F. (1997). Glacial cycles and astronomical forcing. Science, 277(5323), 215-218. https://doi.org/10.1126/science.277.5323.215

Napier, W. M. (2010). Palaeolithic extinctions and the Taurid Complex. Monthly Notices of the Royal Astronomical Society, 405, 1901-1906. 
https://doi.org/10.1111/j.1365-2966.2010.16579.x

Petrie, W. M. F., \& Murray, M. A. (1903). The Osirion at Abydos (Abtu), Egyptian research account-Ninth year. http://ascendingpassage.com/Osirion-atAbydos.htm

Shaltout, M., \& Belmonte, J. A. (2005). On the orientation of ancient Egyptian temples: (1) Upper Egypt and Lower Nubia. Journal for the History of Astronomy, 36(3), 273-298. https://doi.org/10.1177/002182860503600302

Vigato, M. M. (2015). Lost cities of the Mexican Highlands. Uncharted Ruins: Looking for remnants of the lost civilization [blog]. http://unchartedruins.blogspot. com/2015/o6/lost-cities-of-mexican-highlands.html

Wilson, C., \& Flem-Ath, R. (2000). The Atlantis blueprint: Unlocking the ancient mysteries of a long-lost civilization. Delacorte.

Woelfli, W., Baltensperger, W., \& Nufer, R. (2002). An additional planet as a model for the Pleistocene Ice Age. arXiv:physics/0204004

Woodworth, D., \& Gordon, R. G. (2018). Paleolatitude of the Hawaiian hot spot since $48 \mathrm{Ma}$ : Evidence for a Mid-Cenozoic true polar stillstand followed by Late Cenozoic true polar wander coincident with Northern Hemisphere glaciation. Geophysical Research Letters, 45(21), 11632-11,640. https://doi. org/10.1029/2018GLo80787 